\title{
Highly crystalline PCL ultrathin films as thermally switchable biomaterial coatings
}

\author{
Shivam Saretia ${ }^{1,2} \cdot$ Rainhard Machatschek $^{1,2} \cdot$ Andreas Lendlein $^{1,2}$
}

Received: 15 December 2020 / Accepted: 31 January 2021 / Published online: 23 March 2021

(C) The Author(s), under exclusive licence to The Materials Research Society 2021

\begin{abstract}
Semi-crystalline oligomers are explored as functionalized thermoswitchable coatings for modification of biomaterials surface. Highly crystalline oligo( $\varepsilon$-caprolactone) (OCL) films are prepared at the air-water interface by the Langmuir technique, which consist of tightly packed single crystals. Their morphology and melting temperature can be tuned by the chemical structure of the OCL end-groups (hydroxy or methacrylate) and by the crystallization temperature $\left(12\right.$ or $\left.21^{\circ} \mathrm{C}\right)$ as physical parameter. This demand of high crystallite density and adjustable morphology of coatings is not met by conventional methodologies for preparing thin films, e.g., spin coating, spray coating, or solvent evaporation. The high crystallinity reduces the enzymatic degradation rate of the films on both water and solid surfaces. The high density of methacrylate end-groups at the crystal surfaces enables post-functionalization, which was demonstrated using fluorescein dimethacrylate as chemically linked label. The thermoswitching behavior (melting and recrystallization) of fluorescein functionalized, highly crystalline OCL films shows temperature-dependent distribution of the chemically linked fluorescein moieties, which are accumulated on the surfaces of crystals, and homogeneously dispersed when the crystals are molten. Thermally switchable highly crystalline films are relevant for cell substrates modulating adhesion at the biointerface or for coatings as barrier layer influencing the degradation rate.
\end{abstract}

\section{Introduction}

Degradable, semi-crystalline polymers such as poly( $\varepsilon$ caprolactone) (PCL) telechelics are of high technological relevance for switchable biomaterial surfaces to influence cell adhesion at the biointerface and for implant coating as phase-changing lubricant [1]. PCL is established in clinical applications and its enzymatic degradability is welldocumented [2]. In particular, PCL crystals can be used as thermoswitchable units and exhibit, e.g., a change in stiffness and degradability upon melting [3]. The melting temperature of PCL crystals can be adjusted to be very close

Supplementary Information The online version of this article contains supplementary material available at https://doi.org/10. 1557/s43580-021-00020-5.

Andreas Lendlein

andreas.lendlein@hzg.de

1 Institute of Active Polymers and Berlin-Brandenburg Center for Regenerative Therapies, Helmholtz-Zentrum Geesthacht, Kantstr. 55, 14513 Teltow, Germany

2 Institute of Chemistry, University of Potsdam, Karl-Liebknecht-Straße 24-25, 14476 Potsdam, Germany to physiological conditions, producing a polymer layer that changes a broad variety of its functional properties upon application of a small thermal stimulus [1, 4]. Maximizing the effect of the thermal switching requires a high density of crystals having identical orientation. Conventional methods for preparing thin films such as spin coating, spray coating, or solvent evaporation are usually not suited to produce such morphologies [5, 6]. Yet, they require processing conditions involving, e.g., elevated temperatures, centrifugal forces, or organic solvents, which are not well suited for post-functionalization of polymeric biomaterials.

The air-water interface provides an elegant solution for the controlled crystallization of PCL ultrathin films under mild conditions. Using the Langmuir technique, the semicrystalline morphology, including crystal size, number density, thickness, and melting temperature can be precisely adjusted by varying physical parameters such as the aqueous subphase temperature (crystallization temperature), the distance between the barriers, changing the film Mean Molecular Area (MMA), the compression speed or by chemical features such as the OCL telechelic functional end-groups or molecular weight $[7,8]$. The nanostructured polymer films prepared at the air-water interface are transferable on 
different kinds of substrates-hydrophilic or hydrophobic with smooth or rough surfaces [9].

Degradability is a highly relevant function for polymeric biomaterials that is particularly influenced by crystallinity [3]. Here, the Langmuir technique offers the opportunity not only to prepare almost entirely crystalline materials (Fig. 1a), but also to study their degradation behavior and to exactly identify the influence of crystallinity on degradability. It is thereby possible to quantify the extent of adjustment that can be made to the lifetime of a highly crystalline material by thermal switching. In this work, this investigation is carried out using enzymatic catalysis both at the air-water interface and on solid surfaces (Fig. 1b). The exclusion of defects from the polymeric lattice leads to a segregation of chain-ends to the crystal surface [10]. Therefore, single crystals of oligomeric macromolecules are densely covered with chain-ends, which can be converted in a chemical functionalization [11]. Upon melting, the functional end-groups accumulated at the crystalline surface get redistributed in the amorphous phase, resulting in a change of the chemical composition of the material surface. While any chemical functionalization is possible, here a highly crystalline OCL dimethacrylate (OCDME) transferred film was equipped with chemically linked fluorescent moieties (Fig. 1c). The fluorescence emission by fluorescein will be used to distinguish preferable films regions for dye attachment. This dye will also be used to follow the change in the phase morphology during thermally controlled switching.

\section{Experimental}

\section{Materials}

Oligo( $\varepsilon$-caprolactone) diol (OCDOL, trade name CAPA 2803, Solvay Caprolactones, Warrington, U.K.) and oligo( $\varepsilon$ caprolactone) dimethacrylate (OCDME, Sigma Aldrich, Darmstadt, Germany) were used without any further purification. $\mathrm{CDCl}_{3}\left(99.8 \%\right.$, VWR) for ${ }^{1} \mathrm{H}$ NMR and chloroform (HPLC grade, Roth) for GPC were used as the solvents. Bulk material characteristics of OCDOL and OCDME are summarized in Table 1. Details on the bulk characterization methods of OCL samples are given in the supplementary information (Figures S1-S3; Table S1).

\section{(a) HIGHLY CRYSTALLINE FILM PREPARATION \& TRANSFER}

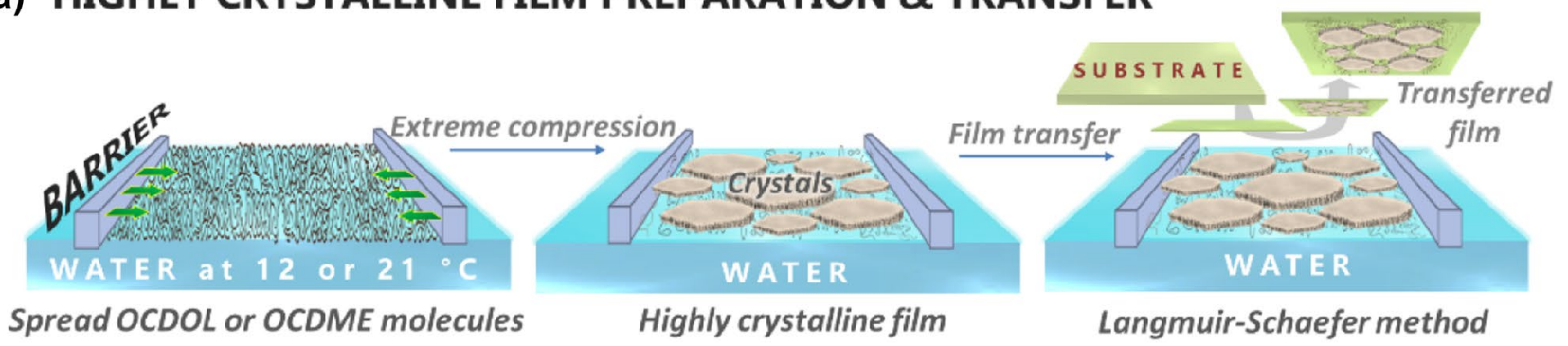

(b) ENZYMATIC DEGRADATION OF HIGHLY CRYSTALLINE VS AMORPHOUS FILMS

WATER SURFACE

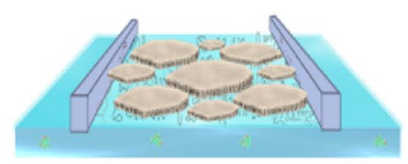

Highly crystalline film

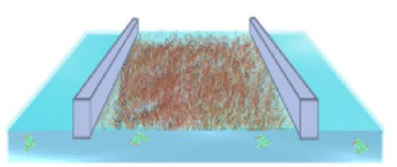

Amorphous film

SILICON SURFACE

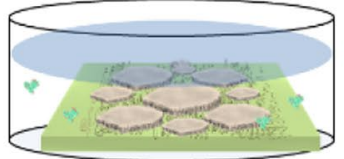

Highly crystalline film

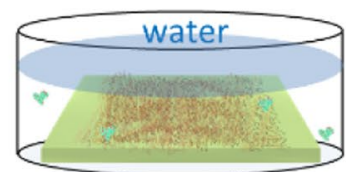

Amorphous film

\section{(c) FUNCTIONALIZATION OF HIGHLY CRYSTALLINE OCDME FILM \& MELTING}

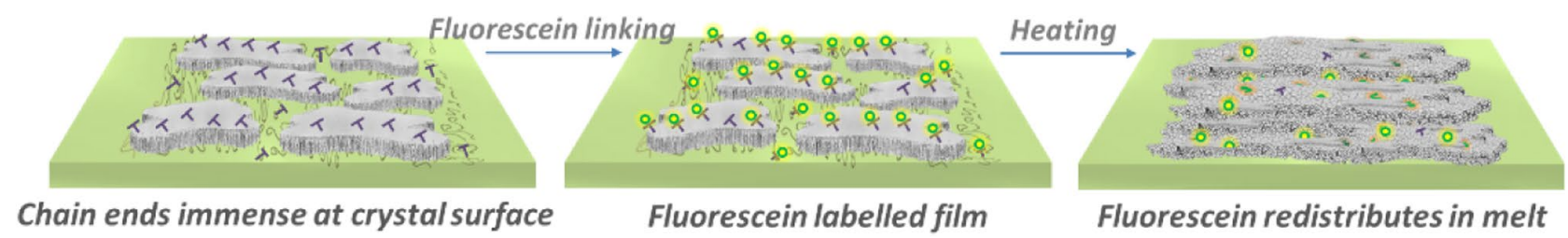

Fig. 1 Schematic representation of the experimental protocol for a preparing, $\mathbf{b}$ transferring and $\mathbf{c}$ investigating highly crystalline OCL ultrathin films 
Table 1 Bulk characteristics of OCL samples determined by GPC, ${ }^{1} \mathrm{H}$ NMR, and DSC

\begin{tabular}{|c|c|c|c|c|c|c|}
\hline Sample & $\begin{array}{l}M_{\mathrm{n}}(\mathrm{GPC})^{\mathrm{a}} \\
{\left[\mathrm{g} \mathrm{mol}^{-1}\right]}\end{array}$ & $\begin{array}{l}M_{\mathrm{n}}\left({ }^{1} \mathrm{H} \mathrm{NMR}\right)^{\mathrm{a}} \\
{\left[\mathrm{g} \mathrm{mol}^{-1}\right]}\end{array}$ & $\begin{array}{l}\text { End-group functionaliza- } \\
\text { tion }\left({ }^{1} \mathrm{H} \mathrm{NMR}\right)^{\mathrm{b}}[\%]\end{array}$ & PDI (GPC) & $\begin{array}{l}T_{\mathrm{c}}(\mathrm{DSC})^{\mathrm{c}} \\
{\left[{ }^{\circ} \mathrm{C}\right]}\end{array}$ & $T_{\mathrm{m}}(\mathrm{DSC})^{\mathrm{c}}\left[{ }^{\circ} \mathrm{C}\right]$ \\
\hline OCDOL & 8300 & 7900 & $95 \pm 3$ & 1.2 & $29 \pm 4.5$ & $56 \pm 4$ \\
\hline OCDME & 5900 & 4300 & $85 \pm 2$ & 1.2 & $18 \pm 4.5$ & $39 \pm 4.5$ \\
\hline
\end{tabular}

${ }^{\mathrm{a}}$ The shift of molecular weights to higher values in GPC than ${ }^{1} \mathrm{H}$ NMR can be related to fractionation of molecules by precipitation

${ }^{\mathrm{b}}$ End-group functionalization determined by ${ }^{1} \mathrm{H}$ NMR analysis was within the limits of experimental error of signal integration. The $M_{\mathrm{n}}$ determined previously via quantification of hydroxy group concentration (OH-value) by potentiometric titration is $8000 \mathrm{~g}^{\mathrm{mol}}{ }^{-1}$. [12]

${ }^{c}$ The temperature range of $T_{\mathrm{c}}$ and $T_{\mathrm{m}}$ was estimated by extrapolation of slopes of exotherms and endotherms from DSC measurements

A Milli-Q Gradient A-10 water purification system (Millipore, Darmstadt, Germany, 18.2 MU cm, toc $<4$ ppb) provided water for the aqueous subphase in Langmuir trough and for dissolution of the lipase enzyme (Pseudomonas cepacia; Sigma, Darmstadt, Germany; $\left.0.007 \mathrm{mg} \cdot \mathrm{mL}^{-1}\right)$ used for degradation experiments. Ethanol (HPLC grade; Bernd Kraft, Duisburg, Germany) and chloroform (HPLC grade, Roth, Karlsruhe, Germany) were used for cleaning of Teflon trough surface, or preparing OCL chloroform solution.

\section{Preparation of OCL film at air-water interface by Langmuir technique}

Details on the Langmuir technique setup and the Teflon trough purification for investigation of films at air-water interface are described in supplementary information (see supplementary information; Table S2). Visualization of OCL film crystallization at the air-water interface was performed by Brewster Angle Microscopy (BAM; ellipsometer nanofilm_ep3, Accurion, Göttingen, Germany) equipped with a high performance CCD camera, a $10 \times$ magnification lens with a maximum lateral resolution of $2 \mu \mathrm{m}$ and a $658 \mathrm{~nm}$ class IIIB laser source. To prepare highly crystalline OCL films at the air-water interface, chloroform solution $\left(0.2-0.3 \mathrm{mg} \cdot \mathrm{mL}^{-1}\right)$ of OCDOL or OCDME was spread dropwise on the surface of water in the Langmuir trough using a micro syringe (Hamilton Co., Reno, NV, USA) such that the OCL film Mean Molecular Area (MMA) was above $7 \AA^{2}$. After a waiting time of $30 \mathrm{~min}$ for chloroform evaporation, the OCL film was symmetrically compressed to an MMA of $\sim 2 \AA^{2}$ at a barrier speed of $10 \mathrm{~mm} \cdot \mathrm{min}^{-1}$.

\section{Enzymatic degradation of OCL films on water surface}

Enzymatic degradation of highly crystalline OCL films was compared with amorphous (melted) OCL films on water surface. The amorphous film at the air-water interface was prepared by melting the highly crystalline OCL film (crystallized at MMA $\sim 2 \AA^{2}$ ) at temperatures $\geq 49{ }^{\circ} \mathrm{C}$. Thereafter, the aqueous subphase was cooled to $46{ }^{\circ} \mathrm{C}$, where recrystallization still does not occur and the film was in the amorphous state. Before degrading the highly crystalline OCL film, the subphase was also heated to $46^{\circ} \mathrm{C}$, where the OCL crystals do not melt. Hence, both highly crystalline and amorphous OCL films at MMA $\sim 2 \AA^{2}$ were degraded at a constant temperature of $46{ }^{\circ} \mathrm{C}$ at the air-water interface. Enzymatic degradation was carried out at constant surface pressure, where lipase solution was inserted into the subphase and $A_{\mathrm{t}} A_{0}{ }^{-1}$, as function of time, was recorded where $\mathrm{A}_{0}$ was the initial surface area occupied by the OCL film at $t=0$ and $A_{\mathrm{t}}$ was the film surface area after a certain degradation time period $t$.

\section{Enzymatic degradation of OCDOL films on silicon surface}

For enzymatic degradation of OCL films on solid surfaces, highly crystalline OCDOL films (crystallized at MMA $\sim 2 \AA^{2}$ ) and amorphous (melted) OCDOL films were transferred to silicon substrates (Plano GmbH, Wetzlar, Germany) by the Langmuir-Schaefer method. They were immersed in enzyme solution at $37 \pm 0.5^{\circ} \mathrm{C}$ which was continuously stirred to avoid enzyme accumulation at the air-water interface. The change in film morphology was observed by optical microscopy and AFM (see supplementary information; Table S3).

\section{OCDME films fluorescein labeling, melting, and recrystallization}

The transferred, highly crystalline OCDME films were reacted with fluorescein dimethacrylate (Sigma, Darmstadt, Germany; $0.5 \mathrm{mg} \cdot \mathrm{mL}^{-1}$; buffer at $\mathrm{pH} 10$; lactone form of fluorescein at acidic or neutral $\mathrm{pH}$ has very low fluorescence while quinoid form at $\mathrm{pH} 10$ is fluorescent active). 
For this purpose, the OCDME films were incubated with fluorescein dimethacrylate solution for $4 \mathrm{~h}$ under presence of UV light ( $2.4 \mathrm{~mW} \mathrm{~cm}{ }^{-2}$; Analytik Jena AG, Germany) and washed thrice with $\mathrm{pH} 10$ buffer. Optical and fluorescent (Carl Zeiss- Filter Set 46) images were acquired during melting and cooling of fluorescence-labeled OCDME crystals in presence of water (Objective Zeiss, W N-Achroplan $63 \mathrm{x} / 0,9)$.

\section{Statistical experimental errors}

OCL films preparation, melting, and degradation experiments were performed at least three times. At the air-water interface, the systematic error range of the surface pressure sensor is $\pm 0.3 \mathrm{mN} \cdot \mathrm{m}^{-1}$ and $\pm 0.2^{\circ} \mathrm{C}$ for the temperature sensor. At the air-water interface, the experimental error during spreading of polymer solution and environmental impurities like dust particles can lead to irregularities in OCL film MMA measurements by $\pm 1 \AA^{2}$. The instrumentation error of rate of heating or cooling at the air-water or air-solid interface is less than $\pm 0.5^{\circ} \mathrm{C} \mathrm{min}{ }^{-1}$. The systematic error of the melting temperature at the air-water interface is given as a temperature range between the points where the first signs of melting and complete melting in the BAM were observed.

\section{Results and discussion}

The spread OCL molecules at the air-water interface were compressed to low MMA by the Langmuir trough barriers. The related compression isotherms (MMA versus surface pressure) are shown in Fig. 2a for two different temperatures of the aqueous subphase. The compression isotherm of both OCDOL and OCDME films is at slightly lower surface pressure values at the lower aqueous subphase temperature of $12{ }^{\circ} \mathrm{C}$, suggesting an influence of temperature on crystallization process at the air-water interface. The crystallization during compression (Fig. 2a) of the OCL films at the air-water interface is visualized by Brewster angle microscopy (Fig. 2b), which shows that the OCL crystals get located closer to each other with increasing compression (Fig. 2b:1-3) and therefore the overall crystallinity increases. At the same time, the amorphous phase represented by the dark region, which surrounds the crystals (Fig. 2b:1), gradually disappears during film compression (Fig. 2b:3). Either new crystals are formed or the existing crystals grow in their lateral size when folded chains further add to their growth front.

At a threshold MMA range ( $\left.M M A_{\text {high crys }}\right)$ between 3.0 and $2.0 \AA^{2}$, which is accompanied with a sudden shoot up of surface pressure (Fig. 2a), the Brewster angle microscopic image (Fig. 3a-d) shows the appearance of highly crystalline OCDOL or OCDME films at the air-water interface at different aqueous subphase temperatures $\left(12\right.$ or $\left.21^{\circ} \mathrm{C}\right)$. At $M M A_{\text {high crys, }}$, the OCL crystals (bright regions; Fig. 3a-d) are tightly packed and rather fixed, i.e., no longer change their lateral position on the water surface, whereas the amorphous regions (dark regions; Fig. 3a-d) are minimal implying that the film is highly crystalline. In terms of morphology, the OCDOL crystal size on water at $12{ }^{\circ} \mathrm{C}$ is smaller than OCDOL crystals at $21^{\circ} \mathrm{C}$ (Fig. 3a-b; Table 2). The OCL crystal shape is influenced by the OCL functional end-group as well. The hydroxy-terminated OCL (OCDOL) forms crystals with smoother edges while OCL dimethacrylate (OCDME) forms crystals with wavy edges. Therefore, highly crystalline films comprised of OCL crystals with different shapes and size are prepared by exploiting OCL functional end-groups (hydroxy or methacrylate) and aqueous subphase temperature (Fig. 3a-d; Table 2) at $M M A_{\text {high crys }}$.

For investigating thermoswitching function, i.e., melting and recrystallization of highly crystalline OCL films at the air-water interface, the OCL samples were compressed
Fig. 2 OCDOL and OCDME film compression isotherms a at the air-water interface at different aqueous subphase temperatures $\left(12\right.$ or $\left.21^{\circ} \mathrm{C}\right)$. Acquired BAM images $\mathbf{b}$ during compression of OCDOL film on aqueous subphase at $21^{\circ} \mathrm{C}$ at the air-water interface (scale bar: $100 \mu \mathrm{m}$ )
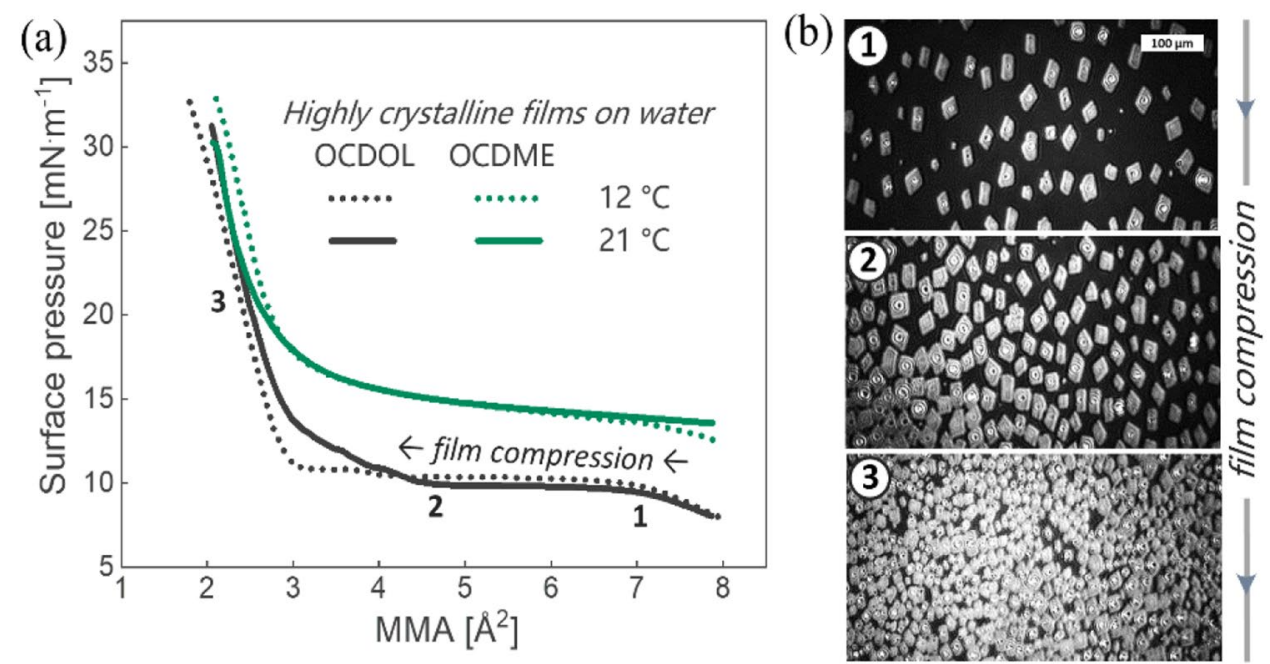
Fig. 3 Acquired BAM images (scale bar: $100 \mu \mathrm{m}$ ) of highly crystalline OCDOL $(\mathbf{a}, \mathbf{b})$ and OCDME films (c, d) (MMA $2 \AA^{2}$ ) at the air-water interface at different aqueous subphase temperatures $\left[12^{\circ} \mathrm{C}\right.$ (a, c) or $\left.21{ }^{\circ} \mathrm{C}(\mathbf{b}, \mathbf{d})\right]$
Table 2 Morphological and thermal characteristics of highly crystalline OCL films (MMA 2 $\AA^{2}$ ) at the air-water interface
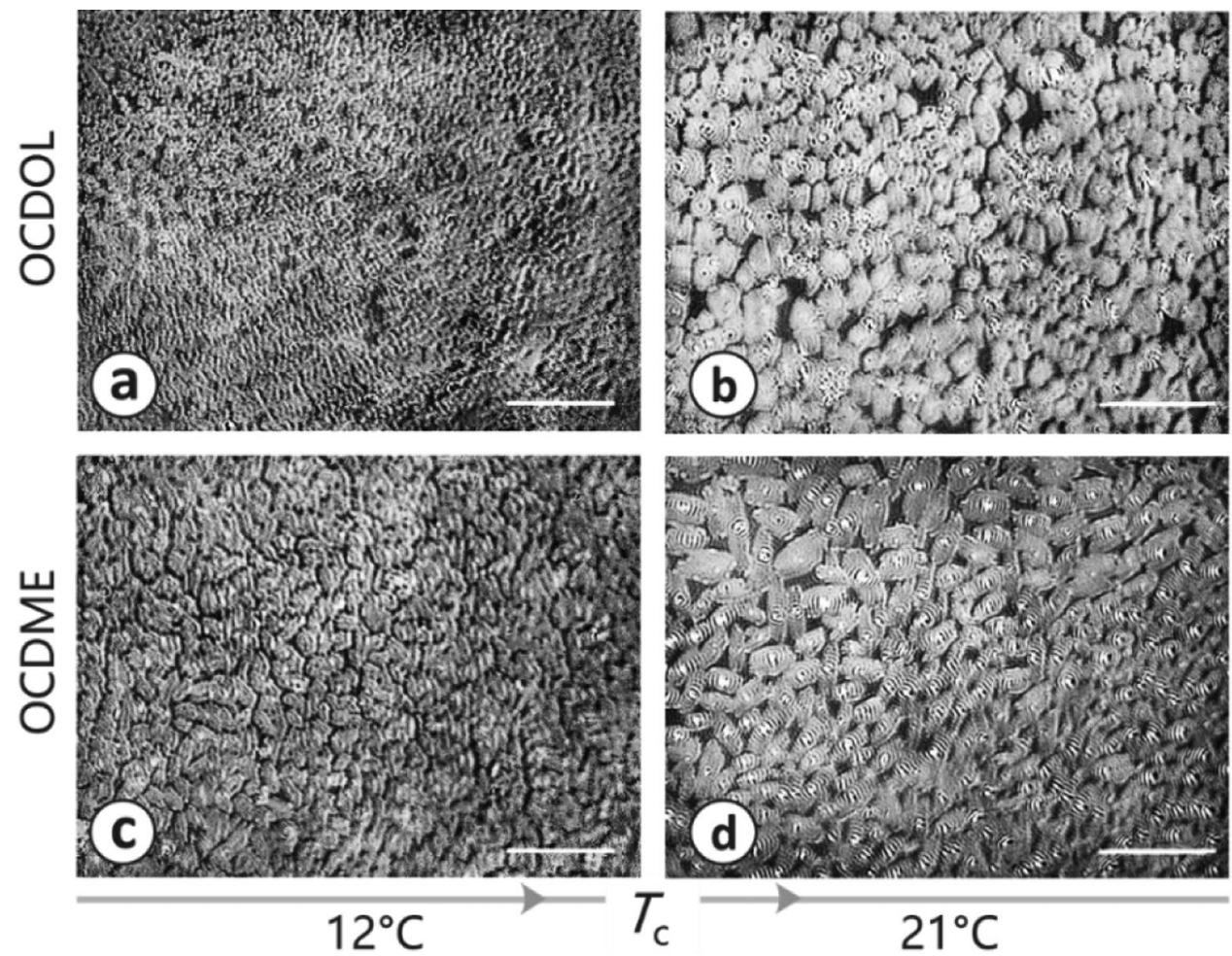

\begin{tabular}{llllll}
\hline OCL sample & $T_{\mathrm{c}}{ }^{\mathrm{a}}\left[{ }^{\circ} \mathrm{C}\right]$ & Crystals morphology & $T_{\mathrm{monset}}{ }^{\mathrm{a}}\left[{ }^{\circ} \mathrm{C}\right]$ & $T_{\mathrm{m} \text { end }}{ }^{\mathrm{a}}\left[{ }^{\circ} \mathrm{C}\right]$ & $T_{\text {rec }}{ }^{\mathrm{a}}\left[{ }^{\circ} \mathrm{C}\right]$ \\
\hline OCDOL & 12 & Smooth edges; $\sim 10 \mu \mathrm{m}$ (Fig. 3a) & 33 & 48 & 44 \\
& 21 & Smooth edges; $\sim 30-60 \mu \mathrm{m}$ (Fig. 3b) & 48 & 50 & 44 \\
OCDME & 12 & Wavy edges; $\sim 20-50 \mu \mathrm{m}$ (Fig. 3c) & 34 & 43 & 39 \\
& 21 & Wavy edges; $\sim 30-60 \mu \mathrm{m}$ (Fig. 3d) & 47 & 49 & 43 \\
\hline
\end{tabular}

The statistical error of $T_{\mathrm{c}}, T_{\mathrm{m} \text { onset }}$ and $T_{\mathrm{m} \text { end }}$ is $\pm 0.5^{\circ} \mathrm{C}$ and of $T_{\text {rec }}$ is $\pm 1.5^{\circ} \mathrm{C}$ to MMA of $2 \AA^{2}$, and after a waiting time of 10 min, the underlying aqueous subphase was heated. By observing the film under BAM, the onset of melting ( $T_{\mathrm{m} \text { onset }} ;$ crystals begin to melt), endpoint of melting ( $T_{\mathrm{m} \text { end }}$; clear BAM image without any crystals), and recrystallization temperature ( $T_{\text {rec }}$; appearance of crystalline structures during cooling) were identified (Table 2).

The highly crystalline OCL films melt under physiologically applicable temperatures and the molten films recrystallize below $45{ }^{\circ} \mathrm{C}$ during cooling of the aqueous subphase (Table 2). Interestingly, the $T_{\mathrm{m} \text { onset }}$ of both OCDOL and OCDME samples is of lower value when the highly crystalline films are prepared at a water temperature of $12{ }^{\circ} \mathrm{C}$. Therefore, the melting temperature range of highly crystalline films at the air-water interface can be adjusted by varying the crystallization temperature $\left(T_{\mathrm{c}}\right)$. Comparing the different molecules, the slightly higher melting temperature range of OCDOL films than OCDME films is attributed to its higher molecular weight.
The enzymatic degradation of bulk polymers proceeds in a surface erosion process, since the enzymes are usually not able to penetrate into the bulk polymer. Therefore, a highly crystalline layer at the surface could serve as a thermally switchable barrier for enzymatic degradation. Here, this effect was investigated for OCDOL and OCDME films at MMA $2 \AA^{2}$, either in highly crystalline or amorphous (melt) state at the air-water interface in the presence of Pseudomonas cepacia lipase both at an identical temperature of $46{ }^{\circ} \mathrm{C}$ (Fig. 4). The highly crystalline OCDOL films are degradable by lipase but with a ca. $3 \times$ slower rate compared to amorphous OCDOL films. The highly crystalline OCDME film degradation is only slightly slower than the OCDME amorphous film degradation. The difference might be explained by the functional end-groups interaction with the enzyme and by the degradation temperature of $46{ }^{\circ} \mathrm{C}$ being much closer to the OCMDE melting temperature range $\left(48 \pm 1{ }^{\circ} \mathrm{C}\right)$. Also, the OCDME crystals could have a larger degree of internal disordering than the OCDOL 


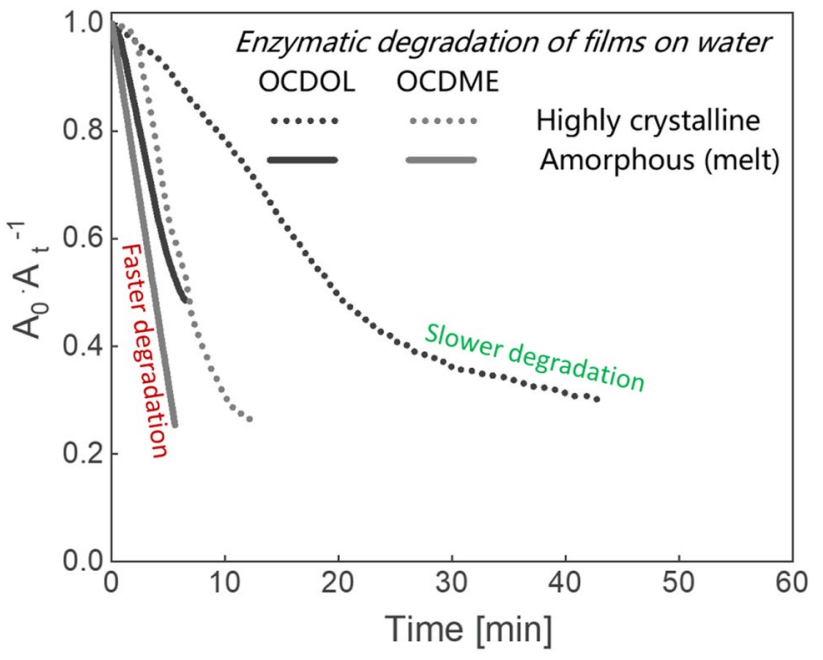

Fig. 4 Enzymatic degradation of highly crystalline OCL films (dotted line) compared to amorphous OCL films (straight line) on the water surface at $46^{\circ} \mathrm{C}$

crystals due to the different chain packing. Altogether, the effect was not very pronounced. Contrary to the hypothesis, crystalline barrier layers are not able to efficiently decelerate the enzymatic degradation of OCL, since the enzymes are catalyzing the degradation of OCL single crystals with great efficiency. Possibly, thicker crystals or multiple layers are required to produce an effective coating stable against enzymatic degradation.

This observation was further substantiated for OCL films on silicon surfaces, when OCDOL films were transferred in their highly crystalline (Fig. 5a:1) or amorphous state (Fig. 5a:2) by the Langmuir-Schaefer method. While the high density of OCDOL crystals (Fig. 5a:1) was maintained upon transfer, the amorphous OCDOL film (Fig. 5a:2) on silicon showed many holes as a consequence of dewetting.

The enzymatic degradation of these transferred OCDOL films (Fig. 5b) proceeded faster than on the water surface (Fig. 4) due to non-identical conditions. The constant stirring and the temperature of $37^{\circ} \mathrm{C}$ (highest activity of enzyme) led to complete dissolution after few minutes (Fig. 5b). While the relative effect was somewhat similar (ca. $2 \times$ slower degradation of highly crystalline vs. amorphous films), the difference in absolute numbers was very small (Fig. 5b).

Bulky end-groups are expelled to the crystal surface during chain packing for crystals formation [11]. The density is inversely proportional to molecular weight and hence very pronounced for oligomers. Therefore, a distinct feature of highly crystalline OCL films is the high density of free unreacted end-groups presented at their surface. Here, this was demonstrated by chemically linking methacrylate end-groups apparent in the OCDME film, which are primarily present at the surface of the crystals, to fluorescein dimethacrylate. The redistribution of the functionalized end-groups during film melting and recrystallization was observed by fluorescence microscopy (Fig. 6). For fluorescein labeling, highly crystalline OCDME film was incubated in fluorescein dimethacrylate solution in the presence of UV light for $4 \mathrm{~h}$. After the photoreaction, the crystals in the OCDME film (Fig. 6e) showed a high degree of fluorescence suggesting successful linking

\section{(a)}
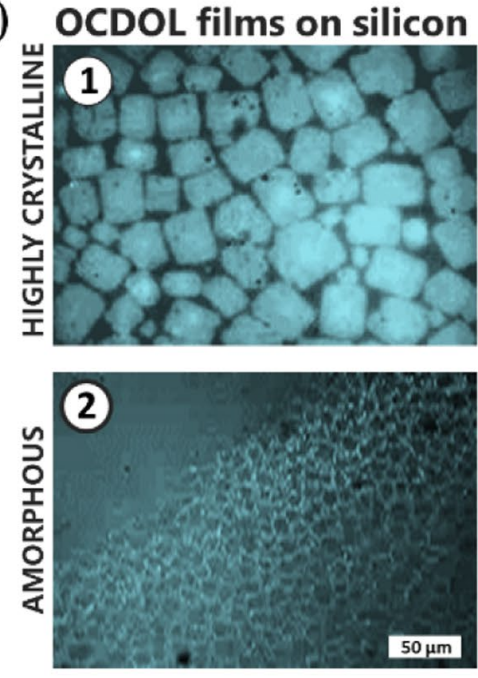

\section{(b)}
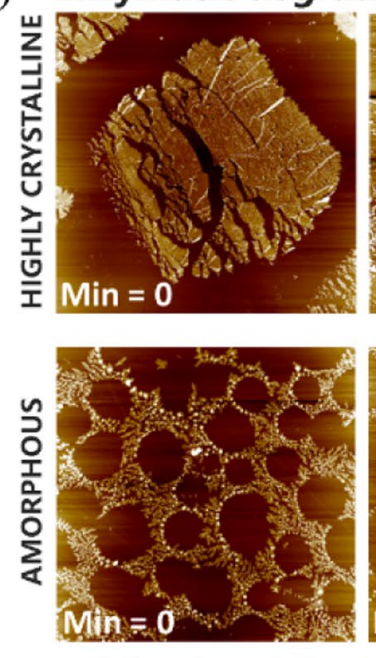

- Before degradation
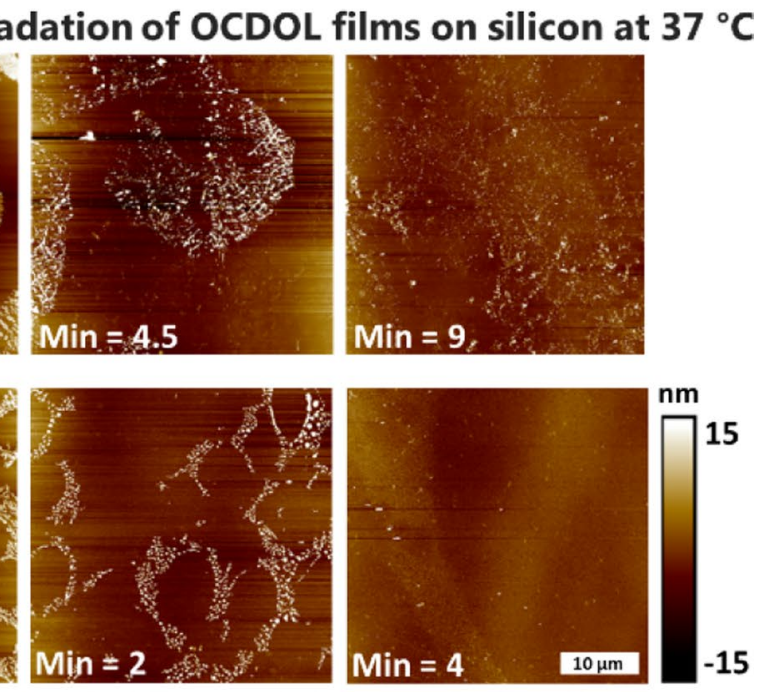

- Partially degraded $\Rightarrow$ Fully degraded
Fig. 5 Optical microscopy images a of transferred highly crystalline OCDOL films (a:1) and amorphous OCDOL films (a:2) on silicon by Langmuir-Schaefer method. High amount of crystals from a highly crystalline film are transferable on silicon with minor fractures (a:1), while transferred amorphous film undergoes dewetting process with holes formation (a:2). AFM images acquired at different intervals of enzymatic degradation b show that the highly crystalline OCDOL films degrade ca. $2 \times$ slower than the amorphous OCDOL film 
Fluorescent labelled OCDME film thermoswitching on silicon

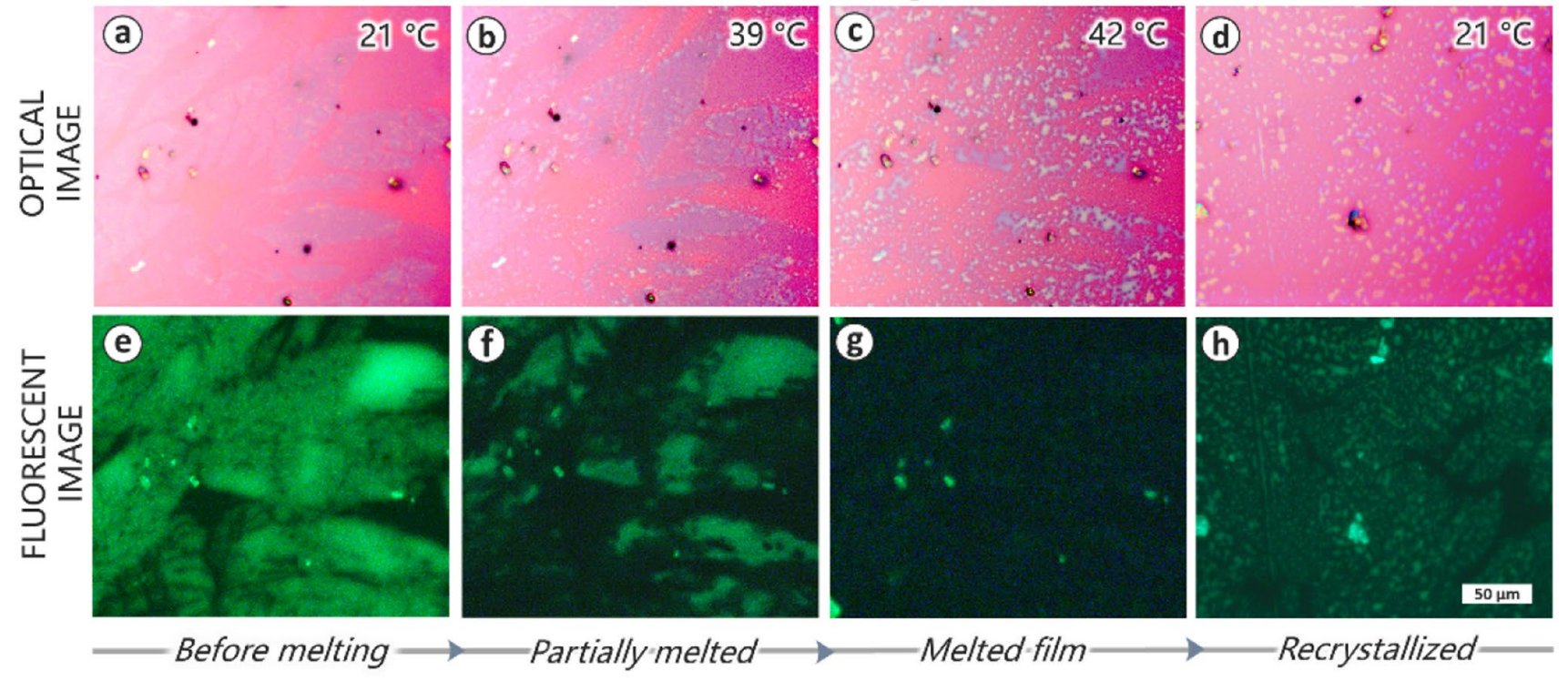

Fig. 6 Optical a-d and fluorescent $\mathbf{e}-\mathbf{h}$ microscopy during thermoswitching of highly crystalline OCME films with chemically linked fluorescein label. a, e: Fluorescein dimethacrylate links to high density of methacrylate end-groups at the OCDME crystals surface evident by their greater degree of fluorescence compared to the surrounding dark amorphous phase. b, c; $\mathbf{f}, \mathbf{g}$ : Melting of highly crystalline OCME films with chemically linked fluorescein is accompanied

of fluorescein to the chain-ends. During the melting of the film in water, (Fig. 6a-c, e-g) the fluorescence intensity of the film is drastically reduced. After cooling, the recrystallized film (Fig. 6h) has a greater fluorescence intensity than the molten film (Fig. 6g). Since the thin polymer layer should be completely transparent for light, a redistribution of fluorescence was expected, but the almost complete quenching of fluorescence intensity in the molten film was unexpected. Some of the polymer chains were certainly dissolved into the heated water, yet the partial recovery of the fluorescence upon recrystallization demonstrates that there must be an additional reason for the quenching in the molten state. For instance, the wettability of the underlying substrate changes with temperature and therefore can influence polymer chains adhesion and the florescence emission [13], while the fluorescein dye is also susceptible to photobleach by environmental oxygen and water $[14,15]$. Nevertheless, the example of fluorescence shows very clearly how the availability and assembly of functional molecules at the surfaces of these highly crystalline films can be switched with temperature. with drastic, uneven lowering of fluorescence intensity in the crystals suggesting redistribution of fluorescein linked OCL end-groups with a possible phase separation. $\mathbf{d}, \mathbf{h}$ : Recrystallized film shows higher fluorescence than the melted film meaning that the fluorescein linked, bulky chain-ends are expelled on the recrystallized structures. The statistical error of temperatures is within limit of $\pm 1.5^{\circ} \mathrm{C}$

\section{Conclusion}

Highly crystalline films of dihydroxy or dimethacrylateterminated linear OCL molecules were prepared at the air-water interface by compressing the molecules to a very low mean molecular area of $\sim 2 \AA^{2}$. Brewster angle microscopy at the air-water interface showed that crystals morphology and thermoswitching behavior (melting and recrystallization) of the highly crystalline OCL films is adjustable by varying the OCL functional end-groups and crystallization temperature. The highly crystalline OCL films were transferred to silicon substrates by the Langmuir- Schaefer method, where transferred OCL crystals are transferable with minor fractures while amorphous films showed dewetting. Highly crystalline OCDOL films are around three times more stable against enzymatic degradation than amorphous OCL films on both water and silicon surfaces; however, they are not very stable in absolute numbers. Fluorescence microscopy of highly 
crystalline films of ODCME functionalized with fluorescein dimethacrylate showed a pronounced change in fluorescence intensity during thermoswitching of the film. This study demonstrates a systematic method for developing highly crystalline OCL films with adjustable morphology, thermoswitching, and degradation behavior. The approach used to prepare the crystals is scalable and can be applied at different biointerfaces. Future investigations could encompass the degradation of these highly crystalline coatings in the absence of enzymes at different $\mathrm{pH}$ values, and the exploration of the films as thermosensitive lubricant for surgical implants to reduce friction in contact with tissue.

Acknowledgments The authors acknowledge the support in characterization by Ms. Manuela Keller for AFM, Dr. Skadi Lau for optical microscopy, Dr. Maria Balk for GPC, Ms. Susanne Schwanz for DSC, and Mr. Olaf Lettau for infrared spectroscopy. This work is financially supported by the Helmholtz Association through program-oriented funding and through Helmholtz Graduate School for Macromolecular Bioscience (MacroBio, VH-GS-503).

Funding Open Access funding enabled and organized by Projekt DEAL.

Data availability Data will be made available on reasonable request.

\section{Compliance with ethical standards}

Conflict of interest The Authors declare that they have no conflicts of interest.

Open Access This article is licensed under a Creative Commons Attribution 4.0 International License, which permits use, sharing, adaptation, distribution and reproduction in any medium or format, as long as you give appropriate credit to the original author(s) and the source, provide a link to the Creative Commons licence, and indicate if changes were made. The images or other third party material in this article are included in the article's Creative Commons licence, unless indicated otherwise in a credit line to the material. If material is not included in the article's Creative Commons licence and your intended use is not permitted by statutory regulation or exceeds the permitted use, you will need to obtain permission directly from the copyright holder. To view a copy of this licence, visit http://creativecommons.org/licenses/by/4.0/.

\section{References}

1. A. Lendlein, O.E.C. Gould, Reprogrammable recovery and actuation behaviour of shape-memory polymers. Nat. Rev. Mater. 4, 116 (2019)

2. H. Sun, L. Mei, C. Song, X. Cui, P. Wang, The in vivo degradation, absorption and excretion of PCL-based implant. Biomaterials 27, 1735 (2006)

3. M. Bartnikowski, T.R. Dargaville, S. Ivanovski, D.W. Hutmacher, Degradation mechanisms of polycaprolactone in the context of chemistry, geometry and environment. Prog. Polym. Sci. 96, 1 (2019)

4. W. Ryu, L. Xiang, T. Jeon, M. Ree, Melt density, equilibrium melting temperature, and crystallization characteristics of highly pure cyclic poly( $\varepsilon$-Caprolactone)s. Polymer 207, 122899 (2020)

5. B. Lotz, T. Miyoshi, S.Z.D. Cheng, 50th Anniversary perspective: polymer crystals and crystallization: personal journeys in a challenging research field. Macromolecules 50, 5995 (2017)

6. Y.-X. Liu, E.-Q. Chen, Polymer crystallization of ultrathin films on solid substrates. Coord. Chem. Rev. 254, 1011 (2010)

7. B. Li, A. Esker, Molar mass dependent growth of poly $(\varepsilon-$ caprolactone) crystals in langmuir films. Langmuir 23, 2546 (2007)

8. S. Saretia, R. Machatschek, B. Schulz, A. Lendlein, Reversible 2D networks of oligo( $\varepsilon$-caprolactone) at the air-water interface. Biomed. Mater. 14, 34103 (2019)

9. K. Ariga, Y. Yamauchi, T. Mori, J.P. Hill, 25th anniversary article: what can be done with the langmuir-blodgett method? recent developments and its critical role in materials science. Adv. Mater. 25, 6477 (2013)

10. S. Yuan, K. Schmidt-Rohr, Immobilized 13C-labeled polyether chain ends confined to the crystallite surface detected by advanced NMR. Sci. Adv. 6, 59 (2020)

11. S. Agbolaghi, S. Abbaspoor and F. Abbasi: A comprehensive review on polymer single crystals-From fundamental concepts to applications. Progress Polym. Sci. (2017).

12. A.-C. Schöne, K. Kratz, B. Schulz and A. Lendlein: Polymer architecture versus chemical structure as adjusting tools for the enzymatic degradation of oligo( $\varepsilon$-caprolactone) based films at the air-water interface. Polym. Degradat. Stab. 131 (2016).

13. R. Gupta, R. Thomas, G.U. Kulkarni, Tunable solid state fluorescence behavior of a methoxy substituted oligo(phenyleneethynylene): influence of cooling rate and surface crystallization. J. Mater. Chem. 22, 19139 (2012)

14. J.R. Saylor, Photobleaching of disodium fluorescein in water. Exp. Fluids 18, 445 (1995)

15. L. Song, E.J. Hennink, I.T. Young, H.J. Tanke, Photobleaching kinetics of fluorescein in quantitative fluorescence microscopy. Biophys. J. 68, 2588 (1995) 